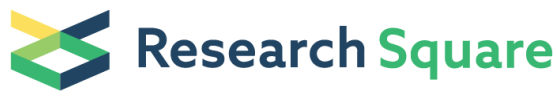 \\ Preprints are preliminary reports that have not undergone peer review. \\ They should not be considered conclusive, used to inform clinical practice, \\ or referenced by the media as validated information.
}

\section{Cardiovascular Health In Pediatric Heart Transplant Patients}

Carmel Bogle ( $\nabla$ carmel.bogle@gmail.com)

Ann and Robert H Lurie Children's Hospital of Chicago https://orcid.org/0000-0002-9230-1497

Amanda M. Perak

Ann \& Robert H. Lurie Children's Hospital of Chicago

Sarah J. Wilkens

University of Louisville School of Medicine

Alaa Aljiffry

Children's Healthcare of Atlanta Egleston Hospital

Karen Rychlik

Ann and Robert H Lurie Children's Hospital of Chicago

John M. Costello

Medical University of South Carolina

Donald Lloyd Jones

Northwestern University Feinberg School of Medicine

Elfriede Pahl

Northwestern University Feinberg School of Medicine

\section{Research article}

Keywords: CVH, glucose, BMI, BP, lipids, NHANES, CKD, CAV

Posted Date: March 10th, 2021

DOI: https://doi.org/10.21203/rs.3.rs-302556/v1

License: (c) (i) This work is licensed under a Creative Commons Attribution 4.0 International License. Read Full License

Version of Record: A version of this preprint was published at The Journal of Heart and Lung Transplantation on April 1st, 2019. See the published version at https://doi.org/10.1016/j.healun.2019.01.1194. 


\section{Abstract \\ Background}

Ideal "cardiovascular health" (CVH)-optimal diet, exercise, nonsmoking, BMI, BP, lipids, and glucose-is associated with healthy longevity in adults. Pediatric heart transplant (HT) patients may be at risk for suboptimal CVH.

\section{Methods}

Single-center retrospective study of HT patients 2003-2014 who survived 1 year post-transplant. Five CVH metrics were collected at listing, 1 , 3 and 5 years post-transplant (diet and exercise were unavailable). $\mathrm{CVH}$ was scored by summing individual metrics: ideal $=2$, intermediate $=1$, and poor $=0$ points; total scores of 8-10 points were considered high (favorable). CVH was compared between HT patients and the US pediatric population (GP) utilizing NHANES 2007-2016. Logistic regression was performed to examine the association of CVH 1 year posttransplant with a composite adverse outcome (death, re-listing, coronary vasculopathy, or chronic kidney disease) 3 years post-transplant.

\section{Results}

We included $110 \mathrm{HT}$ patients (median age at HT: 6 years [range 0.1-21]) and 19,081 NHANES participants. CVH scores among HT patients were generally high at listing (75\%), 1 (74\%), 3 (87\%) and 5 (76\%) years post-transplant and similar to GP, but some metrics (e.g., glucose) were worse among HT patients. Among HT patients, $\mathrm{CVH}$ was poorer with older age and non-Caucasian race/ethnicity. Per 1-point higher CVH score, the demographic-adjusted OR for adverse outcomes was 0.95 (95\% $\mathrm{Cl}, 0.7-1.4)$.

\section{Conclusions}

$\mathrm{HT}$ patients had generally favorable $\mathrm{CVH}$, but some metrics were unfavorable and $\mathrm{CVH}$ varied by age and race/ethnicity. No significant association was detected between $\mathrm{CVH}$ and adverse outcomes in this small sample, but study in a larger sample is warranted.

\section{Background}

Survival after pediatric heart transplant (HT) has improved over time, with more than half of recipients now living at least 17 years after HT (1, 2). Two of the main factors limiting long-term survival among pediatric HT recipients are coronary allograft vasculopathy (CAV) (3) and chronic kidney disease (CKD) (1). Evidence-based preventive strategies are elusive, but observational data in pediatric and adult HT recipients suggest that traditional cardiovascular risk factors, such as dyslipidemia and obesity, are associated with increased risk for these complications (4-6). Previous studies have shown that pediatric HT recipients have elevated levels of total cholesterol (TC) and triglycerides and lower levels of high density lipoprotein (HDL-C) when compared to the general pediatric population (7-10). As dyslipidemia is associated with CAV development in adult HT recipients, afflicted patients may be at increased cardiovascular risk (4, 5, 11-14). CKD, also known to increase mortality and morbidity in both adult and pediatric HT recipients, is also exacerbated by cardiovascular risk factors (15, 16). Despite the continued trend of increased obesity seen in the general pediatric population, with $17 \%$ of children between the ages of $2-19$ years classified as obese and $5.8 \%$ as morbidly obese (17), there have been limited data over the past decade examining multiple cardiovascular risk factors and their implications for HT outcomes in pediatric HT recipients $(5-7,9,13,14,18)$.

In 2010, the American Heart Association (AHA) defined "ideal cardiovascular health (CVH)" as optimal levels of seven metrics (Life's Simple 7): diet, physical activity, nonsmoking, body mass index (BMI), blood pressure (BP), cholesterol, and glucose (19). In the general population, higher levels of $\mathrm{CVH}$, quantified by a CVH "score," have been associated with greater longevity and health-related quality of life and lower healthcare costs (20-22). However, no study has examined CVH or its association with outcomes in the pediatric HT population.

Therefore, we sought to (1) describe the distribution of CVH among pediatric HT recipients, (2) compare these CVH levels with those in the general US pediatric population, and (3) examine the associations of $\mathrm{CVH}$ with a composite adverse outcome including $\mathrm{CKD}$, CAV, re-listing for HT or death.

\section{Methods}

\section{Study Design, Setting and Participants}


We conducted a retrospective single-center study at a large-volume urban pediatric HT center in Chicago, Illinois. Patients who received a primary orthotopic HT from January 1, 2003 to December 31st, 2014 were eligible for the study. January 1. 2003 was selected as the start date to minimize era effects from the widespread change in immunosuppression from cyclosporine to tacrolimus-based therapy after 2000 at our center, as well as routine addition of induction prophylaxis at that time [31]. Patients were excluded from the analyses if they died within the first year after HT, or transferred care out of the institution for >18 months after HT.

For the comparison population, publicly available, de-identified data from the National Health and Nutrition Examination Survey (NHANES) were used. Data from survey cycles 2007-2008 through 2015-2016 were used to match the era of 3- to 5-year follow-up in the HT group (2006-2017), and ages $0-18$ years were included to mirror the age distribution of the HT patients. This comparison group is termed GP (general population).

The Lurie Children's Hospital Institutional Review Board approved the study protocol and the need for written informed consent was waived.

\section{Variables: Cardiovascular Health Metrics and Cardiovascular Health Score}

CVH was assessed via medical record abstraction at multiple time points among HT recipients: time of listing, 1, 3, and 5 years post-HT. For the comparison population, as NHANES is a cross-sectional study, $\mathrm{CVH}$ assessment was at a single time point. Of the seven AHA metrics used to define $\mathrm{CVH}$, diet and physical activity were not consistently available in the electronic medical record for the HT recipient population are therefore not included in the CVH score calculation for either group (HT or GP), as has been done in prior studies using clinical datasets (23, 24). CVH for both the HT sample and GP were defined using the remaining five metrics: smoking status (self-reported), BMI, BP, TC, and fasting blood glucose. In NHANES, CVH metric availability varied by age group as follows: BMI available in all participants, TC available for ages $\geq 6$ years, BP available for ages $\geq 8$ years, smoking status available for ages $\geq 12$ years, and fasting glucose available in a subset of participants $\geq 12$ years old. In addition to the five CVH metrics, lipid fractions including low density lipoprotein cholesterol (LDL-C), high density lipoprotein cholesterol (HDL-C), and triglycerides (TG) among both HT recipients and the GP were secondarily assessed, as these may be relevant for CAV pathogenesis $(5,7,8,14)$.

Individual CVH metrics were each categorized as ideal, intermediate, or poor using AHA's pediatric definitions (Table 1). A composite CVH score was calculated by assigning points to each metric, with 0 points for poor levels, 1 point for intermediate levels, and 2 points for ideal levels, for a total score of 0 to 10 points (Table 1$)$. Composite $\mathrm{CVH}$ scores were then categorized as: $0-4$ points $=$ low, $5-7$ points $=$ moderate, and 8-10 points = high (favorable), as previously reported (25). HT recipients that were placed on a statin for hyperlipidemia or a blood pressure medication had one point subtracted from their respective CVH metric score, as recommended by the AHA CVH definition for "treated" metrics. 
Table 1

Definitions of Cardiovascular Health

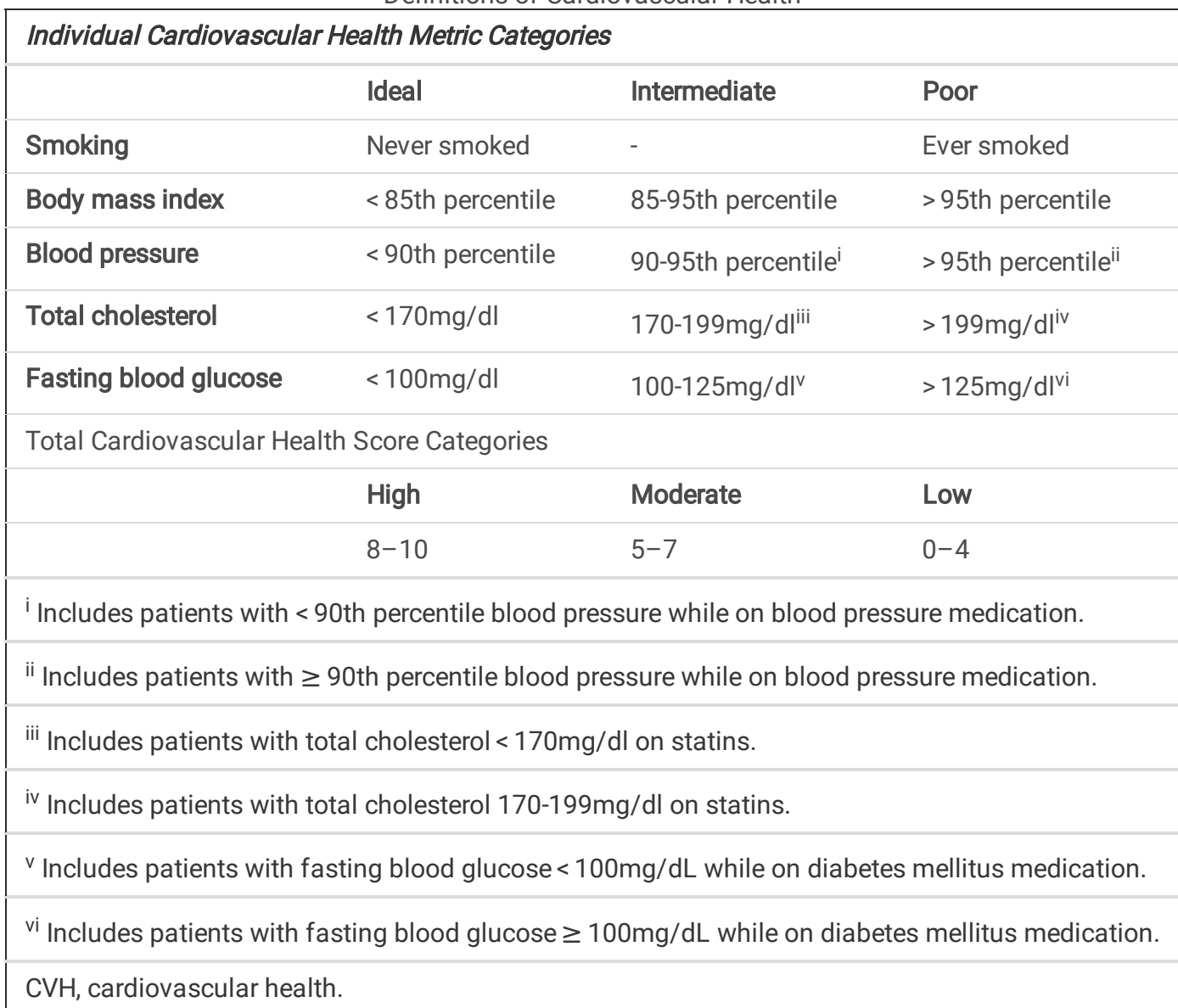

\section{Covariates}

Demographic variables collected included age, sex, and race/ethnicity. For HT recipients, medical history variables were also collected from the medical record, including original cardiac diagnosis, listing date, transplant date, presence and grade of CAV, blood urea nitrogen, creatinine, medications, and diabetes diagnosis.

\section{Adverse Outcomes Among HT Recipients}

Adverse outcomes including CAV, CKD, re-listing and death, were abstracted from patient medical records through 12/31/17. CAV grade was obtained from annual clinical visit notes and cardiac catheterization reports. CAV grading utilized the International Society of Heart and Lung Transplantation (ISHLT) guidelines with modifications from a recent Pediatric Heart Transplant Society (PHTS) study, in which CAV ranges from CAV Grade 0 (not significant) to CAV Grade 3 (severe) (11). CKD, was defined as present if the estimated glomerular filtration rate (eGFR) was $<60 \mathrm{~mL} / \mathrm{min} / 1.73 \mathrm{~m}^{2}$ during routine outpatient visits $(16,26,27)$.

\section{Statistical Methods}

To characterize $\mathrm{CVH}$ among HT recipients, $\mathrm{CVH}$ metrics and CVH scores were calculated and categorized (Table 1). CVH score was counted as a continuous variable, which has been done in multiple large-scale studies $(28-30)$. Continuous variables were reported as mean \pm standard deviation, and categorical variables were reported as counts (percent). CVH was described for the overall group of HT recipients and also for subgroups defined a priori by age at transplant $(0-1,2-5,6-7,8-11$, and $\geq 12$ years), race/ethnicity, and cardiac diagnosis (congenital heart disease or cardiomyopathy). Tests for differences in $\mathrm{CVH}$ among subgroups were considered exploratory, given small subgroup sizes.

To compare CVH between HT recipients and the GP, NHANES survey weights (e.g., fasting weights for glucose analyses) were used to generate accurate population estimates. CVH metrics were compared quantitatively and CVH composite scores (low, moderate, high) were compared between groups qualitatively; due to limitations of statistical inference for comparisons between weighted (NHANES) and un-weighted (HT recipients) data from different populations, statistical significance of differences were not tested.

Finally, to test for an association between $\mathrm{CVH}$ score and adverse outcomes among $\mathrm{HT}$ recipients, univariate and multivariable logistic regression was performed with the exposure $\mathrm{CVH}$ at year 1 post-HT and the outcome adverse HT at year 3 post-HT (year 3 was chosen to 
maximize available sample size). The composite adverse HT outcome included CKD, CAV, re-listing or death. Participants were censored if a non-fatal adverse outcome occurred prior to 1-year post-HT. Both crude and adjusted (for sex, age, transplant age, race/ethnicity and primary cardiac diagnosis) odds ratio for the adverse outcome at year 3 post-HT were calculated, per 1 point higher (more favorable) CVH score at 1 year post-HT.

Statistical analyses were performed using SAS version 9.4 (SAS Institute, Cary, NC). Two-tailed P values $<.05$ were considered statistically significant.

\section{Results}

\section{Study Participants}

A total of 127 patients received a primary HT from January 1st 2003 to December 31st 2014. Seventeen patients were excluded (10 transferred post-HT care to another center for > 18 months; 7 died prior to 1-year post-HT); thus 110 patients were included in the analytic sample. The median age at transplant was 6 years (range $0.1-21$ years), $44 \%$ were female, $43 \%$ were Non-Hispanic Caucasian and $42 \%$ had a primary diagnosis of congenital heart disease. The GP comparison sample included 19,081 children (Table 2).

Table 2

Study Participant Characteristics

\begin{tabular}{|c|c|c|}
\hline & Heart Transplant Recipients, N (\%) & NHANES Sample, N (\%) \\
\hline Total Sample Size & 110 & 19,081 \\
\hline \multicolumn{3}{|l|}{ Sex } \\
\hline Male & $62(56)$ & $9,763(51)$ \\
\hline Female & $48(44)$ & $9,318(49)$ \\
\hline \multicolumn{3}{|l|}{ Ethnicity/Race } \\
\hline Non-Hispanic Caucasian & $63(57)$ & $5,495(29)$ \\
\hline Non-Hispanic African American & $20(18)$ & $4,497(23)$ \\
\hline Hispanic & $26(24)$ & $6,808(36)$ \\
\hline Other/Unknown & $1(1)$ & $2,281(12)$ \\
\hline \multicolumn{3}{|l|}{ Age, Years } \\
\hline $0-1$ & $39(35)$ & $3,600(19)$ \\
\hline $2-5$ & $16(15)$ & $4,335(23)$ \\
\hline $6-11$ & $24(22)$ & $6,267(33)$ \\
\hline $12+$ & $31(28)$ & $4,879(25)$ \\
\hline \multicolumn{3}{|l|}{ Cardiac History } \\
\hline Cardiomyopathy & $64(58)$ & $\mathrm{N} / \mathrm{A}$ \\
\hline Congenital heart disease & $46(42)$ & $\mathrm{N} / \mathrm{A}$ \\
\hline
\end{tabular}

CVH Among HT Recipients

Figure 1 shows the distribution of individual CVH metric levels among HT recipients over time, and eTable 1 provides additional details on mean metric levels and subgroup comparisons. The smoking metric was ideal (never smoked) among $100 \%$ of HT recipients at all points assessed. Most HT recipients had ideal levels of BMI (78-84\%), BP (98-100\%), and TC (79-95\%). Conversely, only about one-half of HT recipients had ideal fasting blood glucose (44-64\%), with 5-20\% having poor glucose levels (the remainder were intermediate; Fig. 1, eTable 1). None of the $\mathrm{CVH}$ metric distributions changed significantly over time, from listing to 5 -years post-HT.

Figure 2A shows the distribution of composite $\mathrm{CVH}$ scores (high, moderate, low) among HT recipients over time, and Table 3 provides additional detail. As with the individual CVH metrics, the majority of HT recipients had high composite CVH score across all time points, with 
mean scores of 7.9 to 8.8 (out of 10) over time. There was no significant difference in composite CVH scores over time.

Table 3

Cardiovascular Health Score in Heart Transplant Recipients, by Time Point and Subgroup

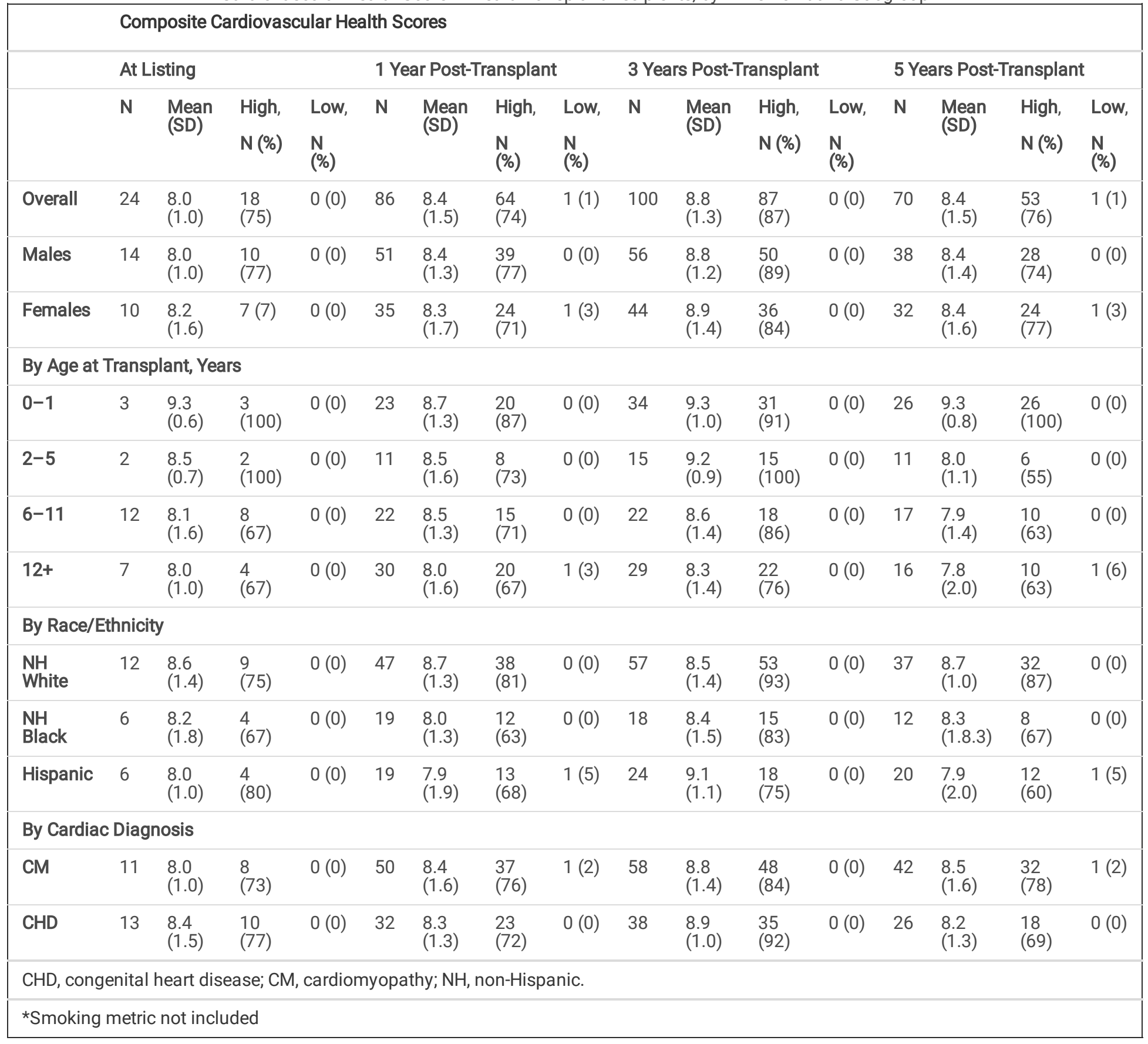

In exploratory subgroup analyses, we explored relationships of patient age, race/ethnicity, sex and diagnosis with CVH and its trends over time post-HT. Younger patients (0-1 years) had higher prevalence of ideal BMI (92-100\%) compared to older patients (12+years: 55-67\%; eTable 2 ), and older patients (12 + years) also had a higher prevalence of a poor composite CVH score (2-6\%) compared with younger patients (Fig. 2B). Non-Hispanic Black HT recipients had a lower prevalence of ideal BMI (58-78\%) compared to the other race/ethnicity groups (6792\%; eTable2), but poor overall CVH scores were most prevalent among the Hispanic subgroup (up to 5\%; Fig. 2B). CVH metric distributions and trends over time post-HT were not substantially different by sex or cardiac diagnosis.

\section{Comparison of CVH between HT Recipients and the General Population}

Figure 3 and eTable 2 show levels of CVH metrics, CVH scores, and lipid fractions among HT recipients and similarly-aged children in the GP. The distribution of composite CVH scores was generally similar between the two groups, as were the distributions of BMI and BP (Fig. 3A). Conversely, the distributions of TC and fasting glucose differed. HT recipients had a higher prevalence of ideal TC compared to the GP (97\% vs $69 \%$; Fig. 3A). Poor TC was present among only $1 \%$ of the HT group, versus $7 \%$ in the GP. Mean levels of TC were also higher in the GP (157 vs. 
120 mg/dL; eTable 2). In secondary analyses of lipid fractions, HT recipients had lower LDL-C (mean 65 vs. 87 mg/dL; eTable 2), but also lower HDL-C (mean 42 vs. 53 mg/dL) when compared to the GP (Fig. 3B). Fasting blood glucose levels were less favorable among HT recipients than GP, with ideal levels present in 52\% versus $77 \%$ and mean levels of 107 and $95 \mathrm{mg} / \mathrm{dL}$, respectively (Fig. 3A, eTable 2).

Association of CVH with Adverse Outcomes Among HT Recipients.

A total of $86 \mathrm{HT}$ patients had full CVH score at 1-year post-transplant with 16 adverse outcomes (11 deaths, 4 CKD, 1 CAV) occurring between 1 and 3 years post-transplant that were included in analysis. Among HT patients with a full CVH score available at 1-year PT and outcomes ascertained at 3-years PT, there was no statistically significant association between year $1 \mathrm{CVH}$ score and the odds for the composite adverse HT outcome in unadjusted (OR $0.87,95 \% \mathrm{Cl} 0.6-1.3)$ or adjusted analysis (OR $0.95,95 \% \mathrm{Cl} 0.7-1.4)$.

\section{Discussion}

To our knowledge, this is the first study to examine $\mathrm{CVH}$ (as defined by the AHA) in the pediatric HT population. In this relatively small analysis of data from $110 \mathrm{HT}$ patients, most HT patients had high (favorable) levels of individual CVH metrics and composite CVH scores with no significant declines over time through 5-years post-HT. In the sub-group analyses older children and minorities held lower $\mathrm{CVH}$ metrics compared to their counterparts. When compared to the US general population of similarly aged children, fasting glucose and HDL-C levels were worse among the HT group, whereas TC and LDL-C were better among the HT group. Among the subset of $86 \mathrm{HT}$ patients with available data, we were unable to detect a significant association between CVH score at 1-year PT and the composite adverse HT outcome (re-listing, death, CAV or CKD) at 3-years PT.

Previous studies examining individual CVH metrics among pediatric HT recipients have been somewhat inconsistent, with some but not all suggesting worse levels of $\mathrm{CVH}$ metrics among $\mathrm{HT}$ recipients compared with the general population. Suboptimal nutrition and a sedentary lifestyle in combination with immunosuppressants and steroid medication are thought to be contributory to worse $\mathrm{CVH}$ metrics after $\mathrm{HT}$ ( 3 , 8 , 29,31 ). Several studies suggested increased risks of obesity, dyslipidemia and a higher incidence of type 2 diabetes post-HT (8, 13, 32-34). For BMI, some studies have suggested increased obesity risks after HT, but a retrospective ISHLT registry study indicated that $8 \%$ of pediatric HT recipients were obese (34), whereas 17\% of children were obese in the US general population based on NHANES data (17). Our estimates of poor BMI (obesity) in HT recipients in the current study are very similar to findings from the ISHLT registry. For BP, limited data exist regarding the incidence of hypertension in pediatric $\mathrm{HT}$ recipients, although a single-center study found that $38 \%$ of pediatric $\mathrm{HT}$ recipients had a systolic blood pressure above the 95th percentile (35). Our single-center study found much lower prevalences of poor BP (hypertension), and further study is needed. Lipids have been more extensively studied among HT recipients. A PHTS study in 2004 (prior to widespread statin use) found that up to $43 \%$ of HT patients had TC $>200 \mathrm{mg} / \mathrm{dL}$ (8), which reflects the immunosuppressant changes over time, as cyclosporine and steroids have been noted to be independent risk factors for dyslipidemia (7) that are less commonly used while statin therapy has been effective in lowering TC and are more integrated in post-HT management in the current era (8). A multi-institutional study in 2006 noted no significant difference in dyslipidemia between the US GP and the pediatric HT population (7), and registry studies have reported similar findings to our study in which the majority of $\mathrm{HT}$ recipients had a normal BMI and TC at time of transplant $(7,34)$. Our more favorable findings for TC and LDL-C likely reflect standard use of statins. However, it is notable that HT recipients had poorer HDL-C levels compared with GP, and this could be due to differences in diet and physical activity levels, which we were not able to measure. For glucose, one study reported a high incidence of $11 \%$ of type 2 diabetes mellitus post-HT after 10 years of follow-up (36). We found poorer glucose levels among HT recipients compared with GP, but in our time frame of follow-up we did not find a high prevalence of poor glucose (diabetes). An important factor to consider in the comparing studies is era and related changes in medication use. For example, studies have shown that when compared to cyclosporine, tacrolimus use was associated with a lower incidence of type 2 diabetes but a higher incidence of dyslipidemia (7, 36). Our study's findings demonstrate not only multiple CVH metrics for HT recipients, but also provide data that is reflective of the current HT immunosuppressant and statin use regimen of this era.

In this single-center study, we did not detect a significant association between CVH score at 1 year post-transplant and adverse outcomes over the following 2 years. Previous studies have shown the association of dyslipidemia with CAV $(5,7,13,31)$ as well as independent associations of diabetes and obesity with adverse outcomes in the pediatric HT population $(8,35,36)$. Given multiple previous studies have shown how various $\mathrm{CVH}$ metrics can contribute to adverse long-term outcomes in the pediatric HT population $(1,5,8,9,12)$, our findings likely reflect lack of power. Future studies with a larger sample size and longer duration of follow-up are needed to further explore associations between suboptimal CVH metrics and adverse HT outcomes. Such studies could also determine whether particular subgroups (e.g. older patients and minorities) remain at significant risk.

\section{Limitations}


There are inherent limitations to a single center retrospective study. First, our findings may not be generalizable to other centers and overall sample size was limited. A larger multicenter cohort would allow meaningful analyses among patient sub-groups, and is planned. Second, we were not able to analyze two of the seven $\mathrm{CVH}$ metrics, diet and physical activity, as this information was not consistently available in medical records. Prospectively capturing these two metrics may be useful. Third, statins were more widely used at our institution during the later period of the study, which could have affected the lipid profiles, we attempted to account for medication use in categorical analyses by subtracting a point from the corresponding CVH score, but we did not adjust continuous lipid levels for statin use. Fourth, the duration of the follow-up available for this study may not have been long enough to capture CAV or late mortality.

\section{Conclusion}

We found that over the first 5-years post-HT, most HT recipients had high CVH scores. However, lipids and glucose were less optimal than the other metrics, and older patients and racial minorities appeared to have lower levels in some metrics. Given prior evidence for the importance of individual $\mathrm{CVH}$ metrics for HT outcomes, further study of $\mathrm{CVH}$ among pediatric HT patients is warranted.

\section{Declarations}

Funding: This study received no funding.

Conflict of Interest: The authors have no conflicts of interest to disclose.

Ethical approval: This article is a retrospective study and does not contain any studies with human participants or animals performed by any of the authors.

Authors' contributions: All authors were involved in the study design, database formation, analysis or manuscript writing of this paper.

\section{References}

1. Copeland H, Razzouk A, Chinnock R, Deming D, Hasaniya N, Bailey L. Pediatric recipient survival beyond 15 post-heart transplant years: a single-center experience. Ann Thorac Surg. 2014;98(6):2145-5.

2. Rossano JW, Cherikh WS, Chambers DC, Goldfarb S, Hayes D, Jr., Khush KK, et al. The International Thoracic Organ Transplant Registry of the International Society for Heart and Lung Transplantation: Twenty-first pediatric heart transplantation report-2018; Focus theme: Multiorgan Transplantation. J Heart Lung Transplant. 2018;37(10):1184-95.

3. Chen AC, Rosenthal DN, Couch SC, Berry S, Stauffer KJ, Brabender J, et al. Healthy hearts in pediatric heart transplant patients with an exercise and diet intervention via live video conferencing-Design and rationale. Pediatr Transplant. 2018:e13316.

4. Pahl E, Naftel DC, Kuhn MA, Shaddy RE, Morrow WR, Canter CE, et al. The impact and outcome of transplant coronary artery disease in a pediatric population: a 9-year multi-institutional study. J Heart Lung Transplant. 2005;24(6):645-51.

5. Kapadia SR, Nissen SE, Ziada KM, Rincon G, Crowe TD, Boparai N, et al. Impact of lipid abnormalities in development and progression of transplant coronary disease: a serial intravascular ultrasound study. J Am Coll Cardiol. 2001;38(1):206-13.

6. Cordero Fort A, Gavira JJ, Alegria-Barrero E, Castano S, Martin A, Ubilla M, et al. Prevalence of metabolic syndrome in heart transplant patients: role of previous cardiopathy and years since the procedure--the TRACA study. J Heart Lung Transplant. 2006;25(10):1192-8.

7. Singh TP, Naftel DC, Webber S, Dupuis J, Chin C, Spicer R, et al. Hyperlipidemia in children after heart transplantation. J Heart Lung Transplant. 2006;25(10):1199-205.

8. Seipelt IM, Crawford SE, Rodgers S, Backer C, Mavroudis C, Seipelt RG, et al. Hypercholesterolemia is common after pediatric heart transplantation: initial experience with pravastatin. J Heart Lung Transplant. 2004;23(3):317-22.

9. Pahl E. Statins in the prevention of transplant coronary artery disease: in pediatric heart recipients. Pediatr Transplant. 2007;11(5):459-60.

10. Pahl E, Crawford SE, Wax DF, Backer CL, Mavroudis C, Gidding S. Safety and efficacy of pravastatin in pediatric heart transplant recipients. J Heart Lung Transplant. 2001;20(2):230.

11. Kindel SJ, Law YM, Chin C, Burch M, Kirklin JK, Naftel DC, et al. Improved Detection of Cardiac Allograft Vasculopathy: A MultiInstitutional Analysis of Functional Parameters in Pediatric Heart Transplant Recipients. J Am Coll Cardiol. 2015;66(5):547-57.

12. Kindel SJ, Pahl E. Current therapies for cardiac allograft vasculopathy in children. Congenit Heart Dis. 2012;7(4):324-35.

13. Chin C, Rosenthal D, Bernstein D. Lipoprotein abnormalities are highly prevalent in pediatric heart transplant recipients. Pediatr Transplant. 2000;4(3):193-9.

14. Valantine H, Rickenbacker P, Kemna M, Hunt S, Chen YD, Reaven G, et al. Metabolic abnormalities characteristic of dysmetabolic syndrome predict the development of transplant coronary artery disease: a prospective study. Circulation. 2001;103(17):2144-52.

Page 8/11 
15. Ojo AO, Held PJ, Port FK, Wolfe RA, Leichtman AB, Young EW, et al. Chronic renal failure after transplantation of a nonrenal organ. N Engl J Med. 2003;349(10):931-40.

16. Feingold B, Zheng J, Law YM, Morrow WR, Hoffman TM, Schechtman KB, et al. Risk factors for late renal dysfunction after pediatric heart transplantation: a multi-institutional study. Pediatr Transplant. 2011;15(7):699-705.

17. Ogden CL, Carroll MD, Lawman HG, Fryar CD, Kruszon-Moran D, Kit BK, et al. Trends in Obesity Prevalence Among Children and Adolescents in the United States, 1988-1994 Through 2013-2014. JAMA. 2016;315(21):2292-9.

18. Sanchez-Lazaro IJ, Sanchez-Gomez JM, Almenar-Bonet L, Martinez-Dolz L, Muno-Giner B, Portoles-Sanz M, et al. Long-term prognostic implications of metabolic syndrome in heart transplant recipients. Transplant Proc. 2011;43(6):2257-9.

19. Lloyd-Jones DM. Cardiovascular risk prediction: basic concepts, current status, and future directions. Circulation. 2010;121(15):1768-77.

20. Lloyd-Jones DM, Hong Y, Labarthe D, Mozaffarian D, Appel LJ, Van Horn L, et al. Defining and setting national goals for cardiovascular health promotion and disease reduction: the American Heart Association's strategic Impact Goal through 2020 and beyond. Circulation. 2010;121(4):586-613.

21. Huffman MD, Capewell S, Ning H, Shay CM, Ford ES, Lloyd-Jones DM. Cardiovascular health behavior and health factor changes (19882008) and projections to 2020: results from the National Health and Nutrition Examination Surveys. Circulation. 2012;125(21):2595-602.

22. Miao C, Bao M, Xing A, Chen S, Wu Y, Cai J, et al. Cardiovascular Health Score and the Risk of Cardiovascular Diseases. PLoS One. 2015;10(7):e0131537.

23. Schiman C, Liu L, Shih YT, Zhao L, Daviglus ML, Liu K, et al. Cardiovascular health in young and middle adulthood and medical care utilization and costs at older age - The Chicago Heart Association Detection Project Industry (CHA). Prev Med. 2019;119:87-98.

24. Allen NB, Zhao L, Liu L, Daviglus M, Liu K, Fries J, et al. Favorable Cardiovascular Health, Compression of Morbidity, and Healthcare Costs: Forty-Year Follow-Up of the CHA Study (Chicago Heart Association Detection Project in Industry). Circulation. 2017;135(18):1693-701.

25. Roth C, Payne PR, Weier RC, Shoben AB, Fletcher EN, Lai AM, et al. The geographic distribution of cardiovascular health in the stroke prevention in healthcare delivery environments (SPHERE) study. J Biomed Inform. 2016;60:95-103.

26. Hollander SA, Montez-Rath ME, Axelrod DM, Krawczeski CD, May LJ, Maeda K, et al. Recovery From Acute Kidney Injury and CKD Following Heart Transplantation in Children, Adolescents, and Young Adults: A Retrospective Cohort Study. Am J Kidney Dis. 2016;68(2):212-8.

27. Feingold B, Brooks MM, Zeevi A, Ohmann EL, Burckart GJ, Ferrell RE, et al. Renal function and genetic polymorphisms in pediatric heart transplant recipients. J Heart Lung Transplant. 2012;31(9):1003-8.

28. Wu S, An S, Li W, Lichtenstein AH, Gao J, Kris-Etherton PM, et al. Association of Trajectory of Cardiovascular Health Score and Incident Cardiovascular Disease. JAMA Netw Open. 2019;2(5):e194758.

29. Enserro DM, Vasan RS, Xanthakis V. Twenty-Year Trends in the American Heart Association Cardiovascular Health Score and Impact on Subclinical and Clinical Cardiovascular Disease: The Framingham Offspring Study. J Am Heart Assoc. 2018;7(11).

30. Xanthakis V, Enserro DM, Murabito JM, Polak JF, Wollert KC, Januzzi JL, et al. Ideal cardiovascular health: associations with biomarkers and subclinical disease and impact on incidence of cardiovascular disease in the Framingham Offspring Study. Circulation. 2014;130(19):1676-83.

31. Rao V. Continuing the battle against cardiac allograft vasculopathy: Does immunosuppression matter or is it a lipid issue? $\mathrm{J}$ Heart Lung Transplant. 2019;38(1):102-3.

32. Armstrong AK, Goldberg CS, Crowley DC, Wei W, Gajarski RJ. Effect of age on lipid profiles in pediatric heart transplant recipients. Pediatr Transplant. 2005;9(4):523-30.

33. Hathout EH, Chinnock RE, Johnston JK, Fitts JA, Razzouk AJ, Mace JW, et al. Pediatric post-transplant diabetes: data from a large cohort of pediatric heart-transplant recipients. Am J Transplant. 2003;3(8):994-8.

34. Kaufman BD, Chuai S, Dobbels F, Shaddy RE. Wasting or obesity at time of transplant does not predict pediatric heart transplant outcomes: analysis of ISHLT pediatric heart transplant registry. J Heart Lung Transplant. 2009;28(12):1273-8.

35. Ryan TD, Zafar F, Siegel RM, Villa CR, Bryant R, 3rd, Chin C. Obesity class does not further stratify outcome in overweight and obese pediatric patients after heart transplantation. Pediatr Transplant. 2018;22(2).

36. Sehgal S, Bock MJ, Louks Palac H, Brickman WJ, Gossett JG, Marino BS, et al. New-onset diabetes mellitus after heart transplantation in children - Incidence and risk factors. Pediatr Transplant. 2016;20(7):963-9.

\section{Figures}




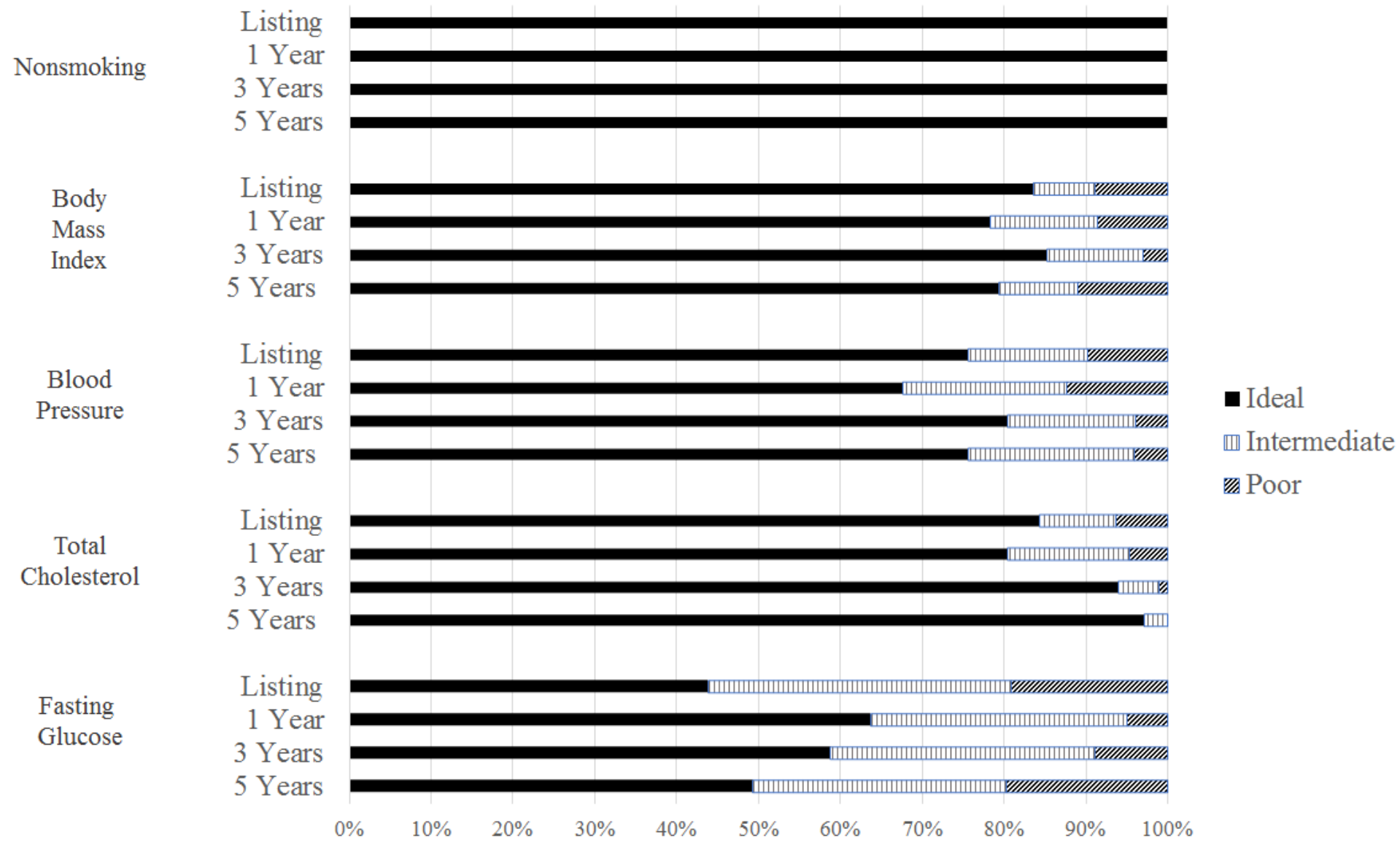

Percentage of Heart Transplant Recipients

\section{Figure 1}

Cardiovascular Health Individual Metric Distributions in Heart Transplant Recipients, By Time Point

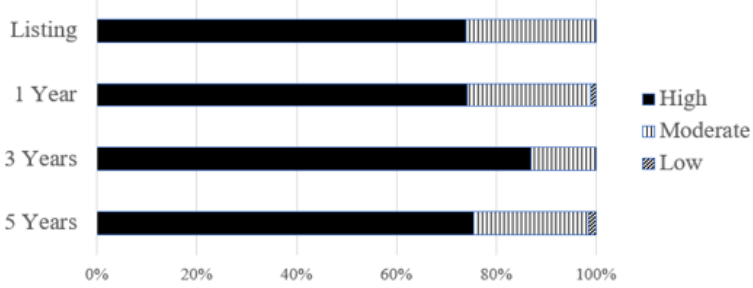

B

Sex
Race/
Ethnicity

Age

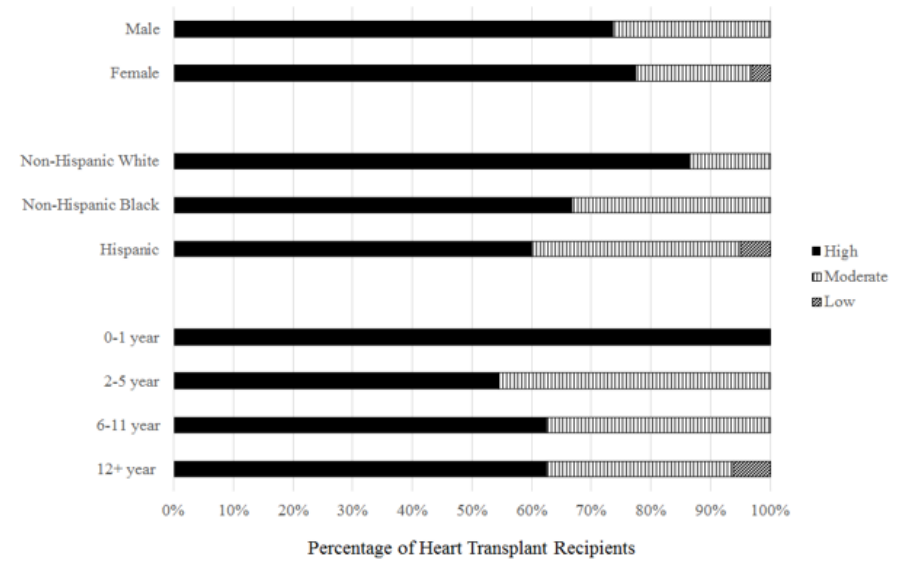

A. Composite cardiovascular health score distributions among all heart transplant recipients, by time point.

B. Composite cardiovascular health score distributions among subgroups of heart transplant recipients at post-transplant Year 5 .

\section{Figure 2}

Composite Cardiovascular Health Score Distributions Among Heart Transplant Recipients, By Time Point and Subgroup 


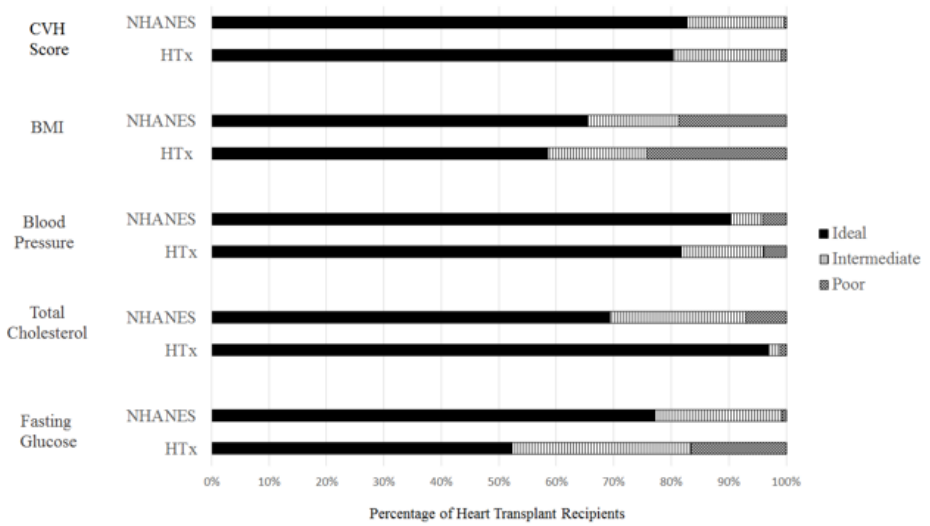

Triglycerides

NHANES

Tx

NHANE

LDL-C

HT

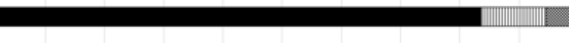

HDL-C

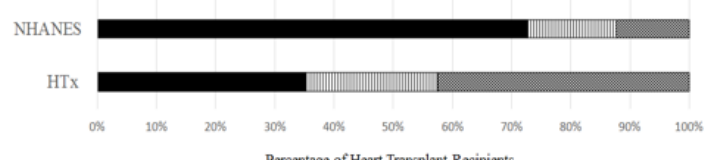

Percentage of Heart Transplant Recipients

A. Cardiovascular health metric distributions are shown among transplant recipients at the latest point of follow up (year 3 or 5 post-transplant), versus the age-, sex-, and race/ethnicity-adjusted general population of United States children, using weighted survey data from the National Health and Nutrition Examination Survey, 2007-2013. See text (Methods) for details. B. Cardiovascular health lipid fractions among transplant recipients at the latest point of follow up (year 3 or 5 post-transplant), versus age-, sex- and race/ethnicity-adjusted general population of United States children, using weighted survey data from the National Health and Nutrition Examination Survey, 2007-2013.

CVH score, cardiovascular health score; BMI, body mass index; HDL-C, high density lipoprotein-calculated; HTx, heart transplant recipients; LDL-C, low density lipoprotein-calculated; NHANES, National Health and Nutrition Examination Survey

\section{Figure 3}

Cardiovascular Health Metrics and Lipid Fractions Among Heart Transplant Recipients (at Years 3-5) Versus the General Population (NHANES)

\section{Supplementary Files}

This is a list of supplementary files associated with this preprint. Click to download.

- eTable1lurie.docx

- eTable2lurie.docx 